\section{Scanning Transmission Electron Microscopy for Critical Dimension Monitoring in Wafer Manufacturing}

\author{
Haifeng Wang*, Jason Fang*, Jason Arjavac**, \\ Rudy Kellner** \\ ${ }^{*}$ Western Digital Corporation, Fremont, CA \\ ${ }^{*}$ FEI Company, Hillsboro, OR \\ Haifeng.Wang@wdc.com
}

Automated scanning transmission electron microscopy (STEM) metrology provides critical dimension (CD) measurements an order of magnitude more precise than comparable scanning electron microscopy (SEM) measurements. New developments in automation now also provide throughput and response time sufficient to support high volume microelectronic manufacturing processes. The newly developed methodology includes automated, focused ion beam (FIB) based sample preparation; innovative, ex-situ sample extraction; and automated metrology. Although originally developed to control the production of thin film magnetic heads for data storage, the technique is fully applicable to any wafer-based manufacturing process.

In many ways, controlling the CDs of thin film heads is even more demanding than controlling CDs of integrated circuits. Each head is formed in a separate die and a thin film head wafer may contain tens of thousands of die (ten to a hundred times more than a typical semiconductor wafer). Current generation heads include features as small as those of the most advanced semiconductor devices. Read and write functions are performed by separate structures within the head. Readers may be less than $100 \mathrm{~nm}$ wide and several tens of nanometers thick, and the transition from giant magneto resistive (GMR) to tunneling magneto resistive (TMR) readers will reduce these dimensions even further. Historically, writers have been several times larger than readers, but the switch from longitudinal magnetic recording (LMR) to perpendicular magnetic recording (PMR) will allow writers with dimensions comparable to the reader.

Similar to the electrical properties of a semiconductor device, the magnetic properties of a thin film head are determined by the geometry and composition of the structure, though the importance of edge effects in magnetic domains makes heads even more sensitive to structural variations.

Like semiconductor devices, heads are fabricated from layers of material patterned by photolithography. Head manufacturing may also include processes, such as ion milling, that are not typically used on integrated circuits. Figure 1 shows top down and cross sectional views of a reader. Important structural characteristics include the dimensions and compositions of all components, the gradients of junction slopes, the thickness of films on the slopes, and many more.
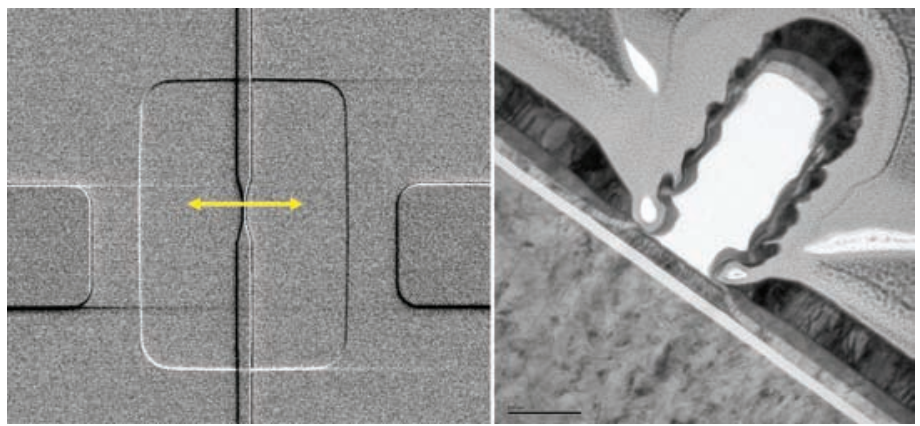

Figure 1. (left) Top-down SEM image showing the patterned reader structure on the wafer; (right) A typical TEM image of cross-section view of the reader structure prepared from a wafer.
Controlling device geometry and film thickness and composition is the key to achieving production consistency and long-term process stability. However, many of the critical structures in a head are buried beneath the surface and are therefore hidden in a top-down view. The most critical surface of the head, known as the air bearing surface (ABS), lies in a plane perpendicular to the wafer surface and is only created when the wafer is cut into individual heads. When assembled, the ABS "flies" on a cushion of air, and holds the now exposed read and write elements in close proximity to the surface of the spinning disk. Final performance of the head may not correlate well with wafer level measurements, and functional testing is not possible until the heads are assembled, many weeks or months after the critical structures were fabricated.

The long delay between wafer processing and functional testing exposes a considerable volume of work-in-progress to risk from an errant process. For this reason DualBeam (FIB/SEM) analysis has become essential to achieving and maintaining profitable yields in head manufacturing operations. By milling a FIB cross section at the precise location of the ABS, engineers can examine the read/write structures immediately after they are formed. Unfortunately, readers and writers are now becoming too small for the SEM based measurements available in a DualBeam system.

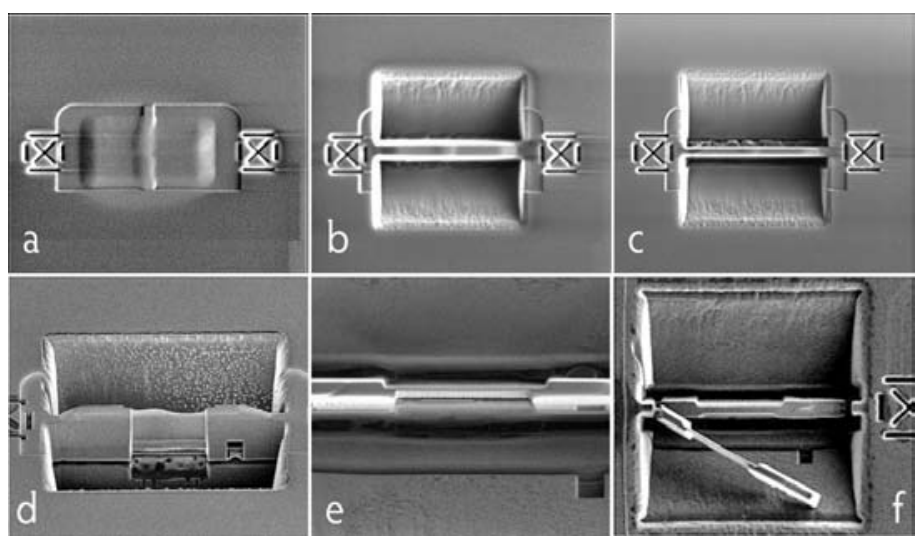

Figure 2. Key FIB sample preparation procedures: (a) milling fiducials, (b) bulk mill, (c) fine polish, (d) bottom release, (e) final polish, and (f) full release.

SEM measurements are limited by the contrast and resolution available in the SEM image. Ultimately, resolution is limited not by the diameter of the scanning beam, but by the size of the volume of interaction - the volume within which the electron beam interacts with the sample to create the imaged signal. In most cases SEMs can deliver resolution down to a few nanometers. Usually the secondary electron (SE) signal offers the best resolution, because low energy SEs can only escape from a very shallow volume. However, the SE signal does not provide the strong material contrast needed to delineate feature boundaries in metrology applications, and is often obscured by the surface topography generated by differential milling in the focused ion beam tool, especially in the case of head wafers.

Transmission electron microscopy (TEM) and STEM are generally capable of resolutions approaching one Angstrom, and in the best case sub Angstrom, but their acceptance for process control applications has been limited by the difficult and time consuming processes needed to prepare the ultra thin samples (less than $100 \mathrm{~nm}$ ) they require. Particularly in STEM, it is the thinness of the sample that permits high resolution by eliminating much of the potential volume of interaction-most incident primary electrons scatter once or not at all as they pass through the sample. Although TEM and STEM are roughly equivalent in resolution capability, STEM is the better candidate for 


\section{Conquering the sub Ångstrom era}

Ultimate performance, stability, and flexibility - FEI's revolutionary Titan is the first and only platform designed to realize the full potential of corrected S/TEM imaging and analysis. With directly interpretable, sub Angstrom image resolution you will, quite literally, see things you've never seen before. Investigate property/structure relationships with atomic resolution. Clearly visualize the interfaces, discontinuities and crystalline defects that are often the key to material properties. Analyze elemental composition, chemical bonding states, electronic band gaps, and more with unprecedented spatial resolution. If you know S/TEM, you know this truly is a revolution.

If your research requires ultimate performance, and your success depends on generating ground breaking new results, call us. We'll be happy to give you an unfair advantage.

See more at fei.com

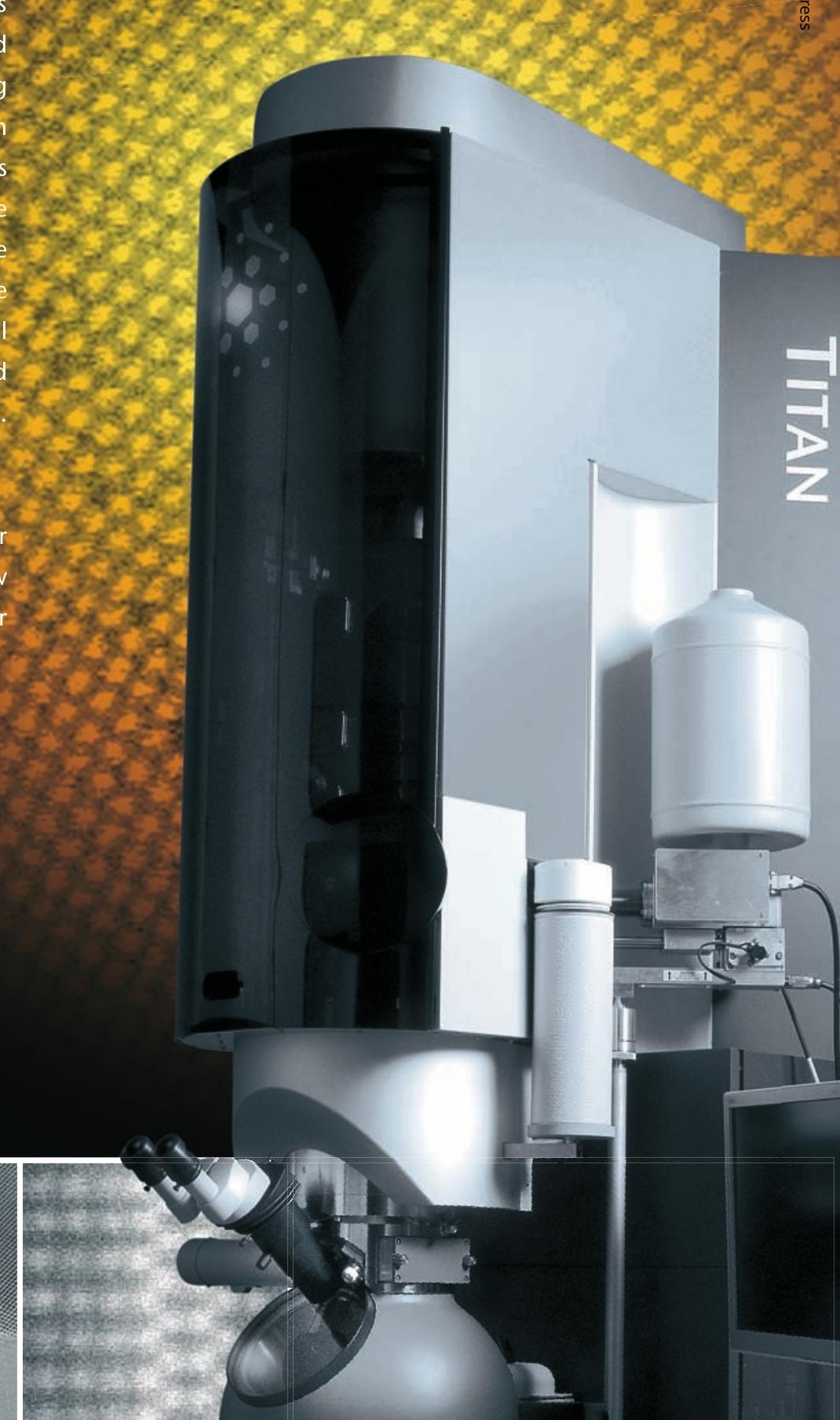




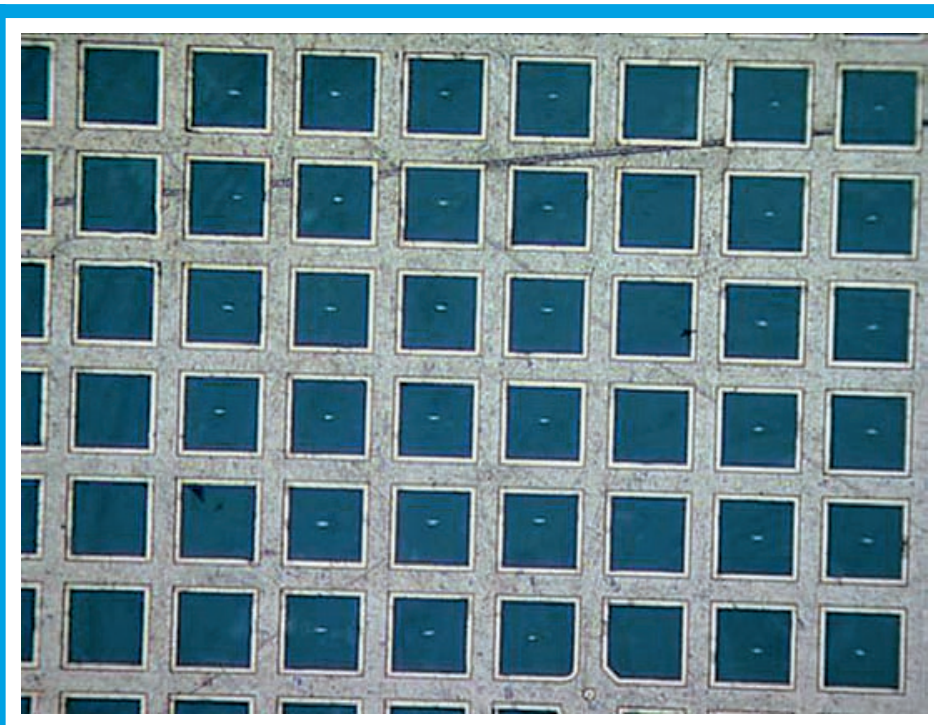

Figure 3. An optical image of a TEM grid loaded with multiple FIB thin sections (the small rectangles centered in grid squares).

thin film head metrology because of the strong material contrast its images exhibit. The phase contrast and diffraction contrast available in TEM images, though useful for other applications, tend to interfere with accurate structural metrology.

Recent developments in sample preparation, sample handling and automated metrology have reduced the difficulty and improved the speed, reliability and reproducibility of STEM structural analysis sufficiently to permit its routine use in controlling high volume manufacturing processes for thin film heads and other microelectronic devices. Automated FIB based sample preparation can cut precisely located thin sections from the full wafer without damaging the remaining heads. Semi-automated ex-situ sample transfer reliably extracts the thin sections from the wafer and positions them on a TEM sample grid. Optimized imaging and automated metrology routines automatically recognize features within the image and measure critical dimensions.

\section{PROCEDURES}

\section{Automated FIB Sample Preparation}

The FIB's ability to position thin cross sections with nanometer accuracy ensures that the extracted section contains the structures that will appear in the ABS. Automation software allows the creation of recipes that control all instrument functions-load lock operation, stage movement, target designation, ion beam milling, and electron beam imaging-from loading the incoming wafers to unloading processed wafers containing fully released thin sections ready for transfer to TEM grids. At the end of the DualBeam process, the system generates a report containing coordinate information for each sample, and exports the data to the ex-situ transfer station for use in the subsequent lift-out process.

Each sample preparation recipe includes three modules: wafer map, site plan, and milling procedures. The wafer map provides a graphic, interactive presentation of the wafer layout with all dimensions and the exact location of every die. The wafer map interfaces with stage control to allow automatic navigation to any die on the wafer. The site plan lists die selected for analysis for a specific type of wafer. The milling procedures specify operating parameters for pattern recognition and FIB milling processes that are performed at each site in the site plan. A typical milling process includes fiducial milling to create reference marks for milling accuracy and drift compensation, fine tuning stage movement, rough trim on both sides of target lamella, bottom release milling, and final polishing to achieve the required thickness and smoothness. Figure 2 shows key steps in the milling process.

\section{Semi-Automated Ex-situ Sample Transfer}

A new, semi-automated process for transferring FIB milled thin sections from the wafer to a TEM sample grid removes another bottleneck in STEM analysis. Conventional methods use a solid glass probe mounted in a micromanipulator and rely on naturally occurring electrostatic forces to attract the section to the probe. They are difficult to control and prone to sample loss during transfer.

The new process incorporates an innovative probe design and computer controlled motorized stage. The new probe, a hollow glass tube, uses vacuum forces to ensure a reliable pick and place operation. A specially shaped tip holds the sample parallel to the grid surface to improve positioning and orientation control when placing the section on the grid. A host computer controls the motorized stage of the transfer station, navigating automatically to sample locations reported by the DualBeam system to pick samples from the wafer, and to specified grid locations to place samples on the grid. The system that tracks the origin (wafer location) of each sample exports this information along with the sample's new location on the grid to the STEM. Electronic data transfer between the DualBeam, transfer station and STEM eliminates the risk of operator errors. Manual interaction is limited to lifting the sample out of the wafer after it has been automatically positioned under the optical microscope, and releasing the sample on the automatically positioned TEM grid. The material of the probe and its coating, the size of the tip and bore, and the strength and flow rate of the vacuum can all be manipulated to accommodate samples of varying size and composition. Performing the transfer ex-situ (outside the FIB chamber) optimizes FIB utilization in the preparation process.

Automation has dramatically improved the speed, precision, and reliability of the transfer process. It provides additional improvements in throughput and efficiency by permitting the placement of a large number of samples on a single grid, with each sample similarly oriented. Multiple samples per grid reduces the number of STEM load cycles. Similar orientations allow the STEM operator to adjust tilt for all samples in a single operation. The approach simplifies sample management by allowing all samples from a wafer to be placed on a single grid or grid section. Figure 3 shows a grid loaded with more than 50 samples.

\section{STEM Imaging and Automated Measurement}

Automated measurement procedures use image analysis and feature recognition routines to find edges and intersections in the digitized image. Dimensional measurements are then derived by counting pixels between designated features and multiplying by a calibrated

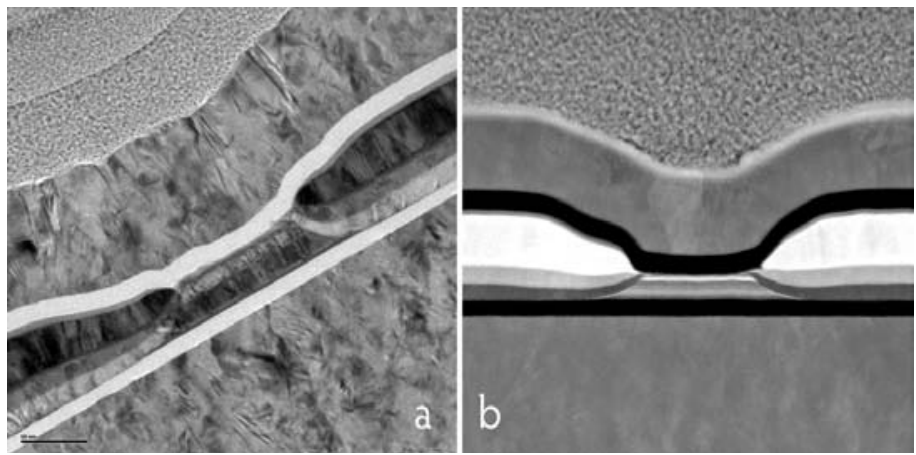

Figure 4. (a) TEM image of a typical GMR reader at high magnification, showing strong strain contrast and diffraction contrast, but ambiguous boundary definition of the layered structure. (b) STEM image of the same structure at comparable magnification, showing clear boundary definition due to the strong atomic number contrast but much reduced diffraction and strain contrast. 


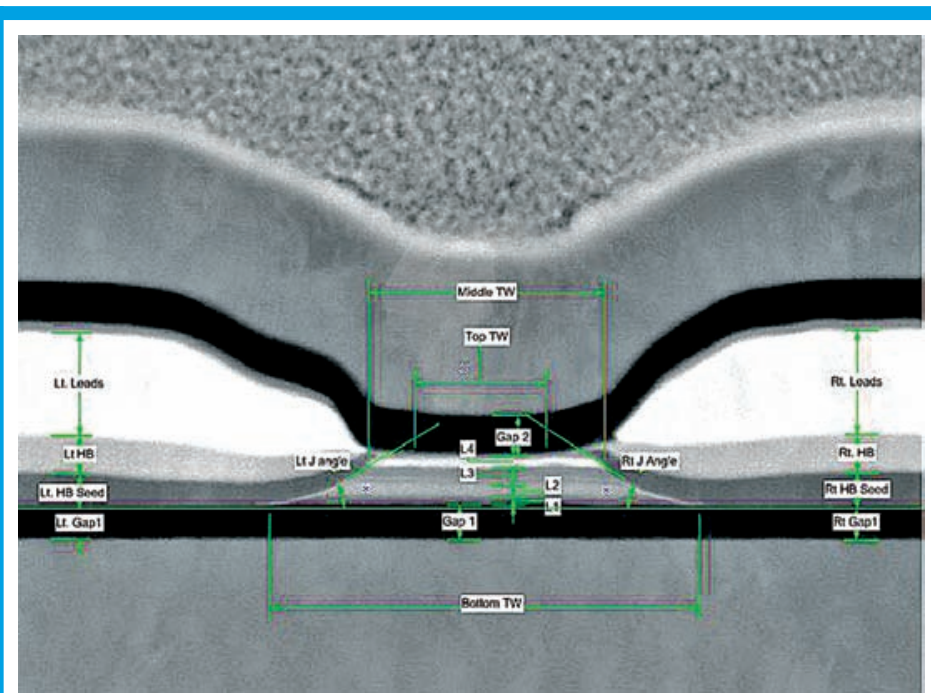

Figure 5. A typical STEM image with labels showing definitions of each measurement on a GMR reader.

conversion factor. The algorithms presume that intensity in the image corresponds to composition in the sample-i.e. material contrast is the dominant contrast mechanism. Although TEM imaging certainly has sufficient spatial resolution for accurate metrology, non-material contrast in TEM images, phase or diffraction contrast, can interfere with the feature recognition and measurement algorithms (Fig 4a). Fortunately, STEM imaging does provide the strong material contrast needed for accurate and reliable automated metrology (Fig $4 \mathrm{~b}$ ).

The S/TEM must be capable of automated operation. The native architecture of the $200 \mathrm{kV}$ Tecnai G2 (FEI Company, Hillsboro, OR, USA) incorporates digital control of all essential instrument functions and provides extensive access for external control and data communications. Automated S/TEM magnification calibration ensures measurement accuracy. Programmable access to internal data structures, such as stage navigation data, permits seamless integration with the DualBeam and lift-out systems. Automated setup and alignment procedures provide fast, easy, consistent operation. Sophisticated STEM automation is essential in achieving the repeatability, reproducibility, reliability and long-term stability required for successful process control.

The STEM analysis procedure is designed to provide consistent STEM image quality with minimal operator intervention. First, the system navigates to each sample on the grid at low magnification. It indexes each sample and creates a filename for STEM images that includes the sample ID transferred from the lift-out system and the grid location. A second round of navigation at final magnification using stored locations is used to fine tune tilt angles for optimal sample

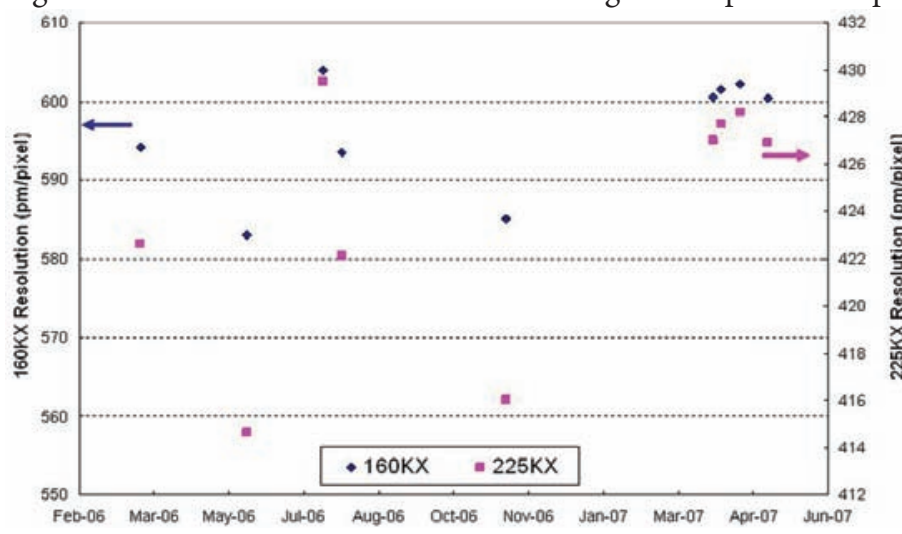

Figure 6. STEM magnification calibration for 2 frequently used magnifications as function of time, illustrating very little magnification variation over time. orientation. Imaging conditions, such as camera length, scan rate, and frame pixels, are adjusted to maximize material contrast. Finally, metrology routines extract and save critical dimensions from the STEM images.(Fig. 5).

\section{RESULTS}

The CD-STEM methodology described above has been deployed for over two years in high volume production of thin film magnetic heads. It consistently provides throughput $5-10$ times greater than conventional methods. Preparation of a $100 \mathrm{~nm}$ thick STEM sample typically takes $20-25$ minutes, including a final polishing step to remove redeposited material. The new ex-situ transfer procedure has a $90 \%$ success rate and reduces the time required for sample transfer to negligible levels. STEM throughput can reach nearly 20 samples per hour over an 8 hour shift.

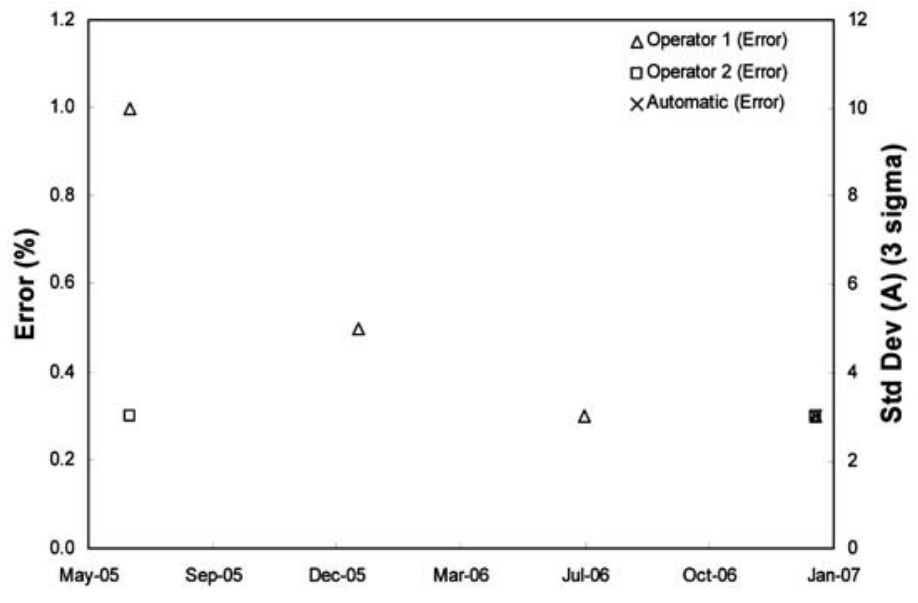

Figure 7. Comparison of metrology precision of a typical feature by various STEM operators with fully automated STEM imaging.

In our experience, automated STEM metrology provides CD measurement precision an order of magnitude better than SEM measurements. Using standards based calibration we have achieved long term measurement consistency of dimensional data better than $98 \%$ (Fig 6). We have also demonstrated measurement precision of $9 \AA$ ( $3 \sigma)$ in a dynamic experiment that included complete load/unload cycles for each measurement. We have identified operator bias associated with the imaging process and are currently developing a fully automated imaging procedure that has shown precision in the $2-4 \AA(3 \sigma)$ range in preliminary tests. (Fig. 7).

\section{CONCLUSION}

Similar to semiconductors, magnetic head manufacturers must now control critical dimensions at the nanoscale. Geometric variability resulting from process variation is increasingly the dominant factor affecting device performance and process yields. The complex edge effects of magnetic domains make heads are even more sensitive than semiconductor devices to structural variability. Controlling threedimensional geometry is absolutely essential to achieving profitable yields from head manufacturing processes. We have described recent developments in STEM metrology that include FIB based sample preparation, ex-situ sample transfer and automated measurement routines. With these advances, automated STEM metrology can provide both the resolution required to accurately characterize nanoscale structures, and the speed, usability, repeatability, reproducibility, reliability and long term stability required for practical process control. This approach is already playing a vital role in our thin film head manufacturing operations and we believe that it can make an equally valuable contribution in any wafer based manufacturing application that requires nanoscale structural process control. 